ARTICLE OPEN

\title{
Risk factors of levodopa-induced dyskinesia in Parkinson's disease: results from the PPMI cohort
}

\author{
Paolo Eusebi $\mathbb{D D}^{1}$, Michele Romoli ${ }^{1}$, Federico Paolini Paoletti ${ }^{1}$, Nicola Tambasco ${ }^{1}$, Paolo Calabresi ${ }^{1,2}$ and Lucilla Parnetti $^{1}$
}

Levodopa-induced dyskinesias (LID) negatively impact on the quality of life of patients with Parkinson's disease (PD). We assessed the risk factors for LID in a cohort of de-novo PD patients enrolled in the Parkinson's Progression Markers Initiative (PPMI). This retrospective cohort study included all PD patients enrolled in the PPMI cohort. Main outcome was the incidence rate of dyskinesia, defined as the first time the patient reported a non-zero score in the item "Time spent with dyskinesia" of the MDS-UPDRS part IV. Predictive value for LID development was assessed for clinical and demographical features, dopamine transporter imaging (DaTscan) pattern, cerebrospinal fluid (CSF) biomarkers (A 342 , total tau, phosphorylated tau, total a synuclein) and genetic risk score for PD. Overall, data from 423 PD patients were analyzed. The cumulative incidence rate of LID was $27.4 \%(95 \% \mathrm{CI}=$ 23.2-32.0\%), with a mean onset time of 5.81 years from PD diagnosis. Multivariate Cox regression analysis showed several factors predicting LID development, including female gender $(\mathrm{HR}=1.61,95 \% \mathrm{Cl}=1.05-2.47)$, being not completely functional independent as measured by the modified Schwab \& England ADL scale ( $\mathrm{HR}=1.81,95 \% \mathrm{Cl}=0.98-3.38)$, higher MDS-UPDRS part III score $(\mathrm{HR}=1.03,95 \% \mathrm{Cl}=1.00-1.05)$, postural instability gait disturbances or intermediate phenotypes $(\mathrm{HR}=1.95,95 \% \mathrm{Cl}=$ 1.28-2.96), higher DaTscan caudate asymmetry index $(\mathrm{HR}=1.02,95 \% \mathrm{Cl}=1.00-1.03)$, higher polygenic genetic risk score $(\mathrm{HR}=$ $1.39,95 \% \mathrm{Cl}=1.08-1.78)$, and an anxiety trait $(\mathrm{HR}=1.02,95 \% \mathrm{Cl}=1.00-1.04)$. In PD patients, cumulative levodopa exposure, female gender, severity of motor and functional impairment, non-tremor dominant clinical phenotype, genetic risk score, anxiety, and marked caudate asymmetric pattern at DaTscan at baseline represent independent risk factors for developing LID.

npj Parkinson's Disease (2018)4:33; doi:10.1038/s41531-018-0069-x

\section{INTRODUCTION}

Parkinson's disease (PD) is a neurodegenerative disorder characterized by motor and non-motor symptoms. ${ }^{1}$ So far, levodopa still stands as the most effective symptomatic treatment for PD. ${ }^{2}$ However, long-term dopamine repletion treatment may lead to motor fluctuations, such as wearing-off and dyskinesias., ${ }^{3,4}$ Several factors participate in the development of motor fluctuations, including loss of dopaminergic neurons in the substantia nigra, changes in pre and post-synaptic striatal activity with chronic pulsatile stimulation of dopamine receptors, ${ }^{5}$ and the daily dosage of levodopa. ${ }^{6}$ Motor fluctuations highly impact on the quality of life of people with $\mathrm{PD}$, representing a major criteria for eligibility to advanced treatments. ${ }^{6}$ Observational studies have shown that more than $50 \%$ of PD patients treated with levodopa for more than 5 years develop levodopa-induced dyskinesia (LID). ${ }^{7}$ Several risk factors for LID have been proposed, including levodopa dosage, ${ }^{8}$ treatment duration, ${ }^{9}$ female gender ${ }^{10}$ and low body weight. ${ }^{11}$ Other factors have been investigated as predisposing to LID, including neuroimaging findings, with conflicting results. ${ }^{12-15}$ It is worth noticing that the available studies are poorly comparable, given the different methodological approaches and follow-up duration, with patients being rarely followed up ever since de novo stage. The Parkinson's Progression Markers Initiative (PPMI) is a large-scale international prospective observational study, started in 2010, designed to identify markers of disease progression in de novo PD patients. Clinical, neuroimaging and
CSF/blood biomarkers are collected yearly. We wanted to define factors predictive of LID development already in de novo stage of PD.

\section{RESULTS}

Cohort characteristics and LID incidence

Baseline demographic and disease characteristics of the cohort divided by the LID - (patients without LID) versus LID+ (patients developing LID) subgroups are presented in Table 1. At the time of data analysis, the median duration of follow-up was 4.6 years (min $0.0 /$ max 6.4). Overall, $109 / 390$ subjects experienced LID (27.9\%, $95 \% \mathrm{Cl} 23.7 \%$ to $32.6 \%$ ). In 33/109 patients experiencing LID, data regarding levodopa treatment and/or LID onset were missing. The median time to LID (Fig. 1) was 3.6 years ( $\min 0.8 / \max 7.1$ ) with an incidence rate of 64 per 1000 person-years.

\section{Analysis of multiple risk factors for time to initiation of} dopaminergic therapy

The average time for initiating a dopaminergic therapy was 1 year. Multivariate Cox regression analysis has shown that a combination of several factors was mildly accurate in predicting the initiation of dopaminergic therapy (Concordance $=0.61,95 \% \mathrm{Cl} 0.57$ to 0.64 ). The Cox proportionality assumption was validated with chi-square test for Schoenfeld residuals (overall $p$-value $=0.089$ ); visual inspection of martingale residuals against individual covariates

\footnotetext{
${ }^{1}$ Neurology Clinic, Department of Medicine, University of Perugia, Ospedale S. Maria della Misericordia, Perugia, Italy and ${ }^{2}$ IRCCS Santa Lucia, Rome, Italy Correspondence: Lucilla Parnetti (lucilla.parnetti@unipg.it)

These authors contributed equally: Paolo Eusebi, Michele Romoli.
}

Received: 26 June 2018 Accepted: 24 October 2018

Published online: 16 November 2018 
Table 1. Baseline demographics, PD characteristics, DaTscan levels, and CSF analytes ( $\beta$-amyloid 1-42 [Ab42], total tau protein [t-Tau], phosphorylated tau protein [p-Tau], and a-synuclein [ $\alpha$-syn]

\begin{tabular}{|c|c|c|c|}
\hline Variable & $\begin{array}{l}\text { LID- (censored } \\
\text { events) } N=289\end{array}$ & $\begin{array}{l}\text { LID }+ \text { (observed } \\
\text { events) } N=109\end{array}$ & $p$-value \\
\hline Age & $62.32 \pm 9.59$ & $60.02 \pm 10.00$ & 0.040 \\
\hline Gender (female) & $92(31.8 \%)$ & $45(41.3 \%)$ & 0.099 \\
\hline Education & $15.43 \pm 2.95$ & $15.87 \pm 2.85$ & 0.170 \\
\hline Disease duration & $0.58 \pm 0.58$ & $0.50 \pm 0.46$ & 0.161 \\
\hline Age at PD diagnosis & $61.74 \pm 9.56$ & $59.52 \pm 9.99$ & 0.047 \\
\hline Family members with PD & $73(25.3 \%)$ & $25(22.9 \%)$ & 0.714 \\
\hline MDS-UPDRS part III Score & $20.25 \pm 8.96$ & $22.60 \pm 8.34$ & 0.015 \\
\hline Hoehn and Yahr & & & 0.264 \\
\hline 1 & $133(46.0 \%)$ & $41(37.6 \%)$ & \\
\hline 2 & $155(53.6 \%)$ & $67(61.5 \%)$ & \\
\hline $3-5$ & $1(0.4 \%)$ & $1(0.9 \%)$ & \\
\hline PIGD Score & $0.21 \pm 0.21$ & $0.27 \pm 0.25$ & 0.017 \\
\hline Tremor Score & $0.49 \pm 0.31$ & $0.49 \pm 0.34$ & 0.964 \\
\hline PD clinical subtype & & & 0.016 \\
\hline Indeterminate & $20(7.1 \%)$ & $13(12.1 \%)$ & \\
\hline PIGD & 47 (16.7\%) & $28(26.2 \%)$ & \\
\hline TD & $215(76.2 \%)$ & $66(61.7 \%)$ & \\
\hline Side most affected & & & 0.139 \\
\hline Left & $114(39.4 \%)$ & $54(49.5 \%)$ & \\
\hline Right & 169 (58.5\%) & $52(47.7 \%)$ & \\
\hline Symmetric & $6(2.1 \%)$ & $3(2.8 \%)$ & \\
\hline Bradykinesia & $238(82.6 \%)$ & $94(87.0 \%)$ & 0.365 \\
\hline Rigidity & $213(74.2 \%)$ & 89 (82.4\%) & 0.115 \\
\hline Tremor & $224(77.5 \%)$ & $84(77.1 \%)$ & 1.000 \\
\hline $\begin{array}{l}\text { Modified Schwab and } \\
\text { England ADL }\end{array}$ & $93.53 \pm 5.82$ & $91.65 \pm 5.89$ & 0.005 \\
\hline UPSIT & $21.96 \pm 7.95$ & $22.33 \pm 9.00$ & 0.708 \\
\hline SCOPA-AUT & $15.13 \pm 6.14$ & $16.06 \pm 6.46$ & 0.197 \\
\hline $\begin{array}{l}\text { Montreal Cognitive } \\
\text { Assessment (MoCA) }\end{array}$ & $27.07 \pm 2.31$ & $27.17 \pm 2.41$ & 0.695 \\
\hline $\begin{array}{l}\text { REM Sleep Behavior } \\
\text { Questionnaire }\end{array}$ & $3.13 \pm 2.11$ & $3.41 \pm 2.06$ & 0.254 \\
\hline Epworth Sleepiness Scale & $32.48 \pm 10.22$ & $5.87 \pm 3.60$ & 0.602 \\
\hline $\begin{array}{l}\text { State-Trait Anxiety } \\
\text { Inventory - State subscore }\end{array}$ & $31.27 \pm 8.98$ & $34.48 \pm 10.16$ & 0.083 \\
\hline $\begin{array}{l}\text { State-Trait Anxiety } \\
\text { Inventory - Trait subscore }\end{array}$ & $46.11 \pm 4.05$ & $34.71 \pm 9.95$ & 0.002 \\
\hline $\begin{array}{l}\text { Geriatric Depression Scale } \\
\text { (GDS-15) }\end{array}$ & $2.13 \pm 2.21$ & $2.72 \pm 2.78$ & 0.046 \\
\hline Genetic risk score & $-1.66 \pm 0.86$ & $-1.44 \pm 0.97$ & 0.052 \\
\hline Mean caudate uptake & 1. $99 \pm 0.55$ & $1.95 \pm 0.57$ & 0.461 \\
\hline $\begin{array}{l}\text { Contralateral caudate } \\
\text { uptake }\end{array}$ & $1.84 \pm 0.54$ & $1.76 \pm 0.56$ & 0.176 \\
\hline Caudate asymmetry index & $17.71 \pm 12.05$ & $22.02 \pm 14.15$ & 0.005 \\
\hline Mean putamen uptake & $0.83 \pm 0.29$ & $0.77 \pm 0.26$ & 0.052 \\
\hline $\begin{array}{l}\text { Contralateral putamen } \\
\text { uptake }\end{array}$ & $0.69 \pm 0.25$ & $0.63 \pm 0.21$ & 0.001 \\
\hline Putamen asymmetry index & $36.16 \pm 24.47$ & $39.79 \pm 25.87$ & 0.209 \\
\hline $\mathrm{A} \beta 42$ & $368.22 \pm 101.55$ & $370.55 \pm 99.36$ & 0.837 \\
\hline t-Tau & $44.84 \pm 18.63$ & $43.19 \pm 16.42$ & 0.396 \\
\hline p-Tau & $15.84 \pm 10.74$ & $14.96 \pm 8.23$ & 0.392 \\
\hline$\alpha-\operatorname{syn}$ & $1813.98 \pm 772.17$ & $1888.06 \pm 790.48$ & 0.406 \\
\hline LEDD & $1347.51 \pm 1255.83$ & $2108.58 \pm 1797.02$ & $<0.001$ \\
\hline Levodopa LEDD & $713.73 \pm 927.79$ & $1491.64 \pm 1595.91$ & $<0.001$ \\
\hline Dopamine Agonists LEDD & $310.30 \pm 491.45$ & $263.42 \pm 415.74$ & 0.344 \\
\hline MAO-B LEDD & $64.59 \pm 71.59$ & $66.68 \pm 73.44$ & 0.800 \\
\hline Amantadine LEDD & $69.75 \pm 160.50$ & $120.64 \pm 225.38$ & 0.033 \\
\hline
\end{tabular}

\section{DISCUSSION}

LID negatively affects the quality of life of patients with PD. Despite extensive research, conflicting results have been gathered regarding modifiable and non-modifiable risk factors for LID development, ${ }^{16}$ with few studies assessing de novo patients. In

supported the linearity hypothesis. The final model included disease duration ( $\mathrm{HR}=0.81,95 \% \mathrm{Cl} 0.66$ to 1.00$)$, Modified Schwab \& England $\mathrm{ADL}$ score $<90$ ( $\mathrm{HR}=1.58,95 \% \mathrm{Cl} 1.09$ to 2.32 ), higher MDS-UPDRS part III ( $\mathrm{HR}=1.02,95 \% \mathrm{Cl} 1.01$ to 1.03$)$, contralateral putamen ( $\mathrm{HR}=0.47,95 \% \mathrm{Cl} 0.30$ to 0.73 ), the polygenic risk score ( $\mathrm{HR}=1.18,95 \% \mathrm{Cl} 1.04$ to 1.34$)$, and the anxiety trait as measured by STAI subscore (HR=1.01, $95 \% \mathrm{Cl} 1.00$ to 1.03$)$.

Analysis of risk factors for LID

The mean time for onset of dyskinesia after the initiation of any dopaminergic therapy was 3.6 years. The predictive value of each variable for investigating the incidence of LID was explored using Cox regression models, with total Levodopa equivalent daily dose (LEDD) included as covariate. Female gender was associated to a greater risk of developing dyskinesia $(\mathrm{HR}=1.79,95 \% \mathrm{Cl} 1.21$ to 2.66, Table 2, Fig. 1). Several clinical characteristics were associated with incidence of dyskinesia such as Modified Schwab \& England ADL $(\mathrm{HR}=0.96,95 \% \mathrm{Cl} 0.93$ to 0.99 , Table 2, Fig. 1), PIGD/ Intermediate phenotype $(\mathrm{HR}=1.75,95 \% \mathrm{Cl} 1.18$ to 2.59 , Table 2, Fig. 1), MDS-UPDRS part III ( $\mathrm{HR}=1.03,95 \% \mathrm{Cl} 1.01$ to 1.05 , Table 2, Fig. 1), and PIGD subtype (HR=3.11,95\% Cl 1.42 to 6.79, Table 2, Fig. 1). Interestingly, several DaTscan measures were associated with a greater risk of dyskinesia such as contralateral putamen (HR $=0.40,95 \% \mathrm{Cl} 0.14$ to 0.84 , Table 2, Fig. 1), caudate asymmetry index ( $\mathrm{HR}=1.02,95 \% \mathrm{Cl} 1.01$ to 1.04 , Table 2, Fig. 1), and putamen asymmetry index ( $\mathrm{HR}=1.01,95 \% \mathrm{Cl} 1.00$ to 1.02 , Table 2, Fig. 1$)$. In addition, onset of dyskinesia was associated with psychiatric features such as depression ( $\mathrm{HR}=1.11,95 \% \mathrm{Cl} 1.04$ to 1.19 , Table 2) and anxiety as measured by STAI-State score $(\mathrm{HR}=1.02,95 \% \mathrm{Cl}$ 1.00 to 1.04 , Table 2) and STAI-Trait score (HR=1.04, 95\% Cl 1.02 to 1.06 , Table 2, Fig. 1). None of the CSF biomarkers showed any predictive power.

Analysis of multiple risk factors for LID

Multivariate Cox regression analysis has shown that a combination of multiple factors was fairly accurate in predicting the onset of dyskinesia (Concordance $=0.74,95 \% \mathrm{Cl} 0.681$ to 0.80 ). The Cox proportionality assumption was validated with chi-square test for Schoenfeld residuals (overall $p$-value $=0.383$ ), and visual inspection of martingale residuals against individual covariates supports the hypothesis of linearity. The final model included female gender $(\mathrm{HR}=1.63,95 \% \mathrm{Cl} 1.06$ to 2.50 , Table 3$), 1000 \mathrm{mg} / \mathrm{d}$ of Levodopa LEDD ( $\mathrm{HR}=1.22,95 \% \mathrm{Cl} 1.08$ to 1.38 , Table 3 ), STAI Trait score (HR $=1.03,95 \% \mathrm{Cl} 1.00$ to 1.05 , Table 3), Modified Schwab \& England ADL (HR $=0.97,95 \% \mathrm{Cl} 0.93$ to 1.01 , Table 3$)$, MDS-UPDRS part III (HR $=1.03,95 \% \mathrm{Cl} 1.00$ to 1.05 , Table 3), PIGD/Intermediate phenotype $(\mathrm{HR}=2.04,95 \% \mathrm{Cl} 1.33$ to 3.13 , Table 3$)$, caudate asymmetry index ( $\mathrm{HR}=1.02,95 \% \mathrm{Cl} 1.00$ to 1.03 , Table 3$)$, and the genetic risk score $(\mathrm{HR}=1.35,95 \% \mathrm{Cl} 1.05$ to 1.74 , Table 3$)$.

Random survival forests for LID

After fitting random survival forests with 1000 trees and missing data imputation, 12 variables were selected using minimal depth criterion leading to an out-of-bag error of $24 \%$. The final model was consistent with the results of multivariate Cox regression in terms of the selected variables. Using random survival forests, also CSF biomarkers entered in the panel of predictive factors (Table 2) for LID development, with a-syn ranking 6th according to the importance of all variables (Fig. 2). 

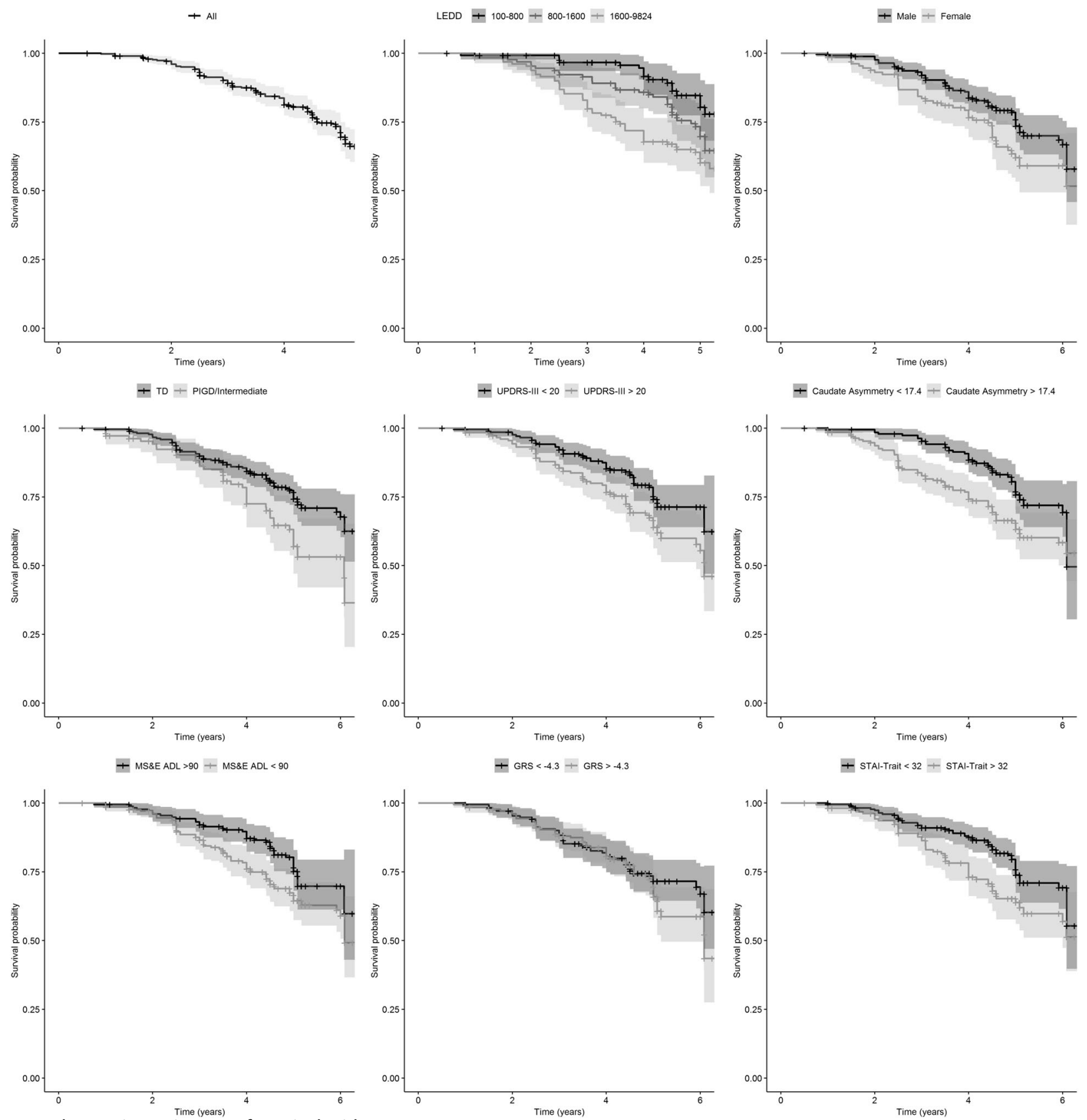

Fig. 1 Kaplan-Meier estimates of survival without LID

this study, evaluating data of de novo PD patients included in the PPMI cohort, we identified a set of seven independent risk factors for LID development that can be taken into consideration already in the very early phase of the disease.

First of all, we confirm that female gender represents a crucial non-modifiable predictive factor for LID, $^{10}$ independently from body weight and genetic factors. ${ }^{10,11,17}$ Second, our results underline that cumulative exposure to levodopa is positively associated with the development of LID, in line with the results deriving from other large cohorts of PD patients. ${ }^{18,19}$ Noteworthy, it is well known that levodopa accelerates the loss of nigrostriatal dopamine nerve terminals, a key pathophysiologic element in the development of dyskinesia. Dyskinesia in Parkinson's disease are associated with changes in long term neuroadaptation and neuronal synaptic plasticity which in turn are linked to dopamine transporters and receptors density, respectively at a presynaptic and a postsynaptic level. ${ }^{6}$ Based on this concept, both positron emission tomography (PET) and single photon emission computed tomography (SPECT) were used to assess changes in neurotransmitter pathways involved in dyskinesia and, subsequently, to identify imaging biomarkers for LID development. Notably, lower dopamine transporter activity in the putamen evaluated by ${ }^{18} \mathrm{~F}$-FP-CIT-PET in de-novo PD patients has been described as significant predictor of LID. ${ }^{13}$ Our results indicate that dopamine deficits in the contralateral putamen in de-novo PD patients is an independent predictor of a shorter time to levodopa initiation. Besides, both putamen asymmetry and caudate asymmetry indices evaluated by $\left[{ }^{123} \mathrm{I}\right] \mathrm{FP}-\mathrm{CIT}-\mathrm{SPECT}$ significantly correlated with the development of dyskinesia, with the caudate asymmetry entering in the multivariate model. These findings reflect previous evidences indicating a positive relationship between the striatal asymmetric index and the magnitude of 
Table 2. Cox regression analysis of individual risk factors adjusted for total LEDD

\begin{tabular}{llll}
\hline Variable & HR & HR $95 \%$ Cl & $p$-value \\
\hline Age & 0.982 & $0.964-1.001$ & 0.063 \\
Gender (female) & 1.794 & $1.212-2.656$ & 0.004 \\
Education & 1.013 & $0.950-1.080$ & 0.695 \\
Disease duration & 0.769 & $0.522-1.133$ & 0.184 \\
Age of PD diagnosis & 0.983 & $0.965-1.002$ & 0.073 \\
Family history of PD & 0.798 & $0.509-1.251$ & 0.325 \\
PIGD/Intermediate vs. TD & 1.745 & $1.178-2.584$ & 0.005 \\
MDS-UPDRS part III Score & 1.028 & $1.007-1.049$ & 0.009 \\
Hoehn and Yahr >1 & 1.409 & $0.952-2.085$ & 0.087 \\
Tremor Score & 0.899 & $0.477-1.697$ & 0.744 \\
PIGD Score & 3.109 & $1.424-6.790$ & 0.004 \\
Mean caudate & 0.814 & $0.563-1.178$ & 0.275 \\
Contralateral caudate & 0.724 & $0.496-1.057$ & 0.094 \\
Caudate asymmetry index & 1.022 & $1.007-1.037$ & 0.003 \\
Mean putamen & 0.529 & $0.246-1.138$ & 0.103 \\
Contralateral putamen & 0.400 & $0.138-0.839$ & 0.019 \\
Putamen asymmetry index & 1.008 & $1.000-1.016$ & 0.043 \\
Modified Schwab and England ADL & 0.956 & $0.927-0.986$ & 0.004 \\
UPSIT & 1.014 & $0.991-1.038$ & 0.239 \\
SCOPA-AUT & 1.016 & $0.987-1.047$ & 0.280 \\
Montreal Cognitive Assessment (MoCA) & 1.001 & $0.921-1.088$ & 0.981 \\
REM Sleep Behavior Questionnaire & 1.045 & $0.953-1.146$ & 0.351 \\
Epworth Sleepiness Scale & 1.005 & $0.950-1.063$ & 0.862 \\
State-Trait Anxiety Inventory - State & 1.018 & $1.001-1.036$ & 0.042 \\
subscore & & & \\
State-Trait Anxiety Inventory - Trait & 1.037 & $1.018-1.056$ & $<0.001$ \\
subscore & & & \\
Geriatric Depression Scale (GDS-15) & 1.111 & $1.037-1.191$ & 0.003 \\
Genetic risk score *100 & 1.200 & $0.961-1.469$ & 0.107 \\
A 342 & 1.001 & $0.999-1.002$ & 0.601 \\
t-Tau & 0.999 & $0.987-1.010$ & 0.802 \\
p-Tau & 0.994 & $0.974-1.015$ & 0.579 \\
$\alpha$-syn & 1.000 & $0.999-1.001$ & 0.367 \\
LEDD & 1.233 & $1.120-1.357$ & $<0.001$ \\
Levodopa LEDD & 1.359 & $1.225-1.508$ & $<0.001$ \\
Dopamine agonists LEDD & 0.693 & $0.427-1.123$ & 0.136 \\
MAO-B LEDD & 0.607 & $0.044-8.458$ & 0.710 \\
Amantadine LEDD & 2.632 & $1.067-6.496$ & 0.036 \\
\hline Hazard ratios with 95\% confidence intervals and $p$-values & \\
\hline
\end{tabular}

response to levodopa. ${ }^{20}$ Thus, PD patients with higher striatal asymmetric index show an increase of both response to levodopa and susceptibility to dyskinesia.

The probability of developing dyskinesia in Parkinson's disease is also influenced by the initial clinical phenotype. Our findings showed that tremor-dominant (TD) phenotype is at lower risk of LID compared to PIGD or Intermediate. Previous studies have already demonstrated that tremor-dominant manifestation at disease onset is associated with a reduced risk of LID compared to rigid-akinetic (RA) phenotype. ${ }^{21}$ The reason of a lower risk of dyskinesia among TD patients may lay in different patterns of nigrostriatal denervation, morphologic lesions of the basal ganglia subregions and pathophysiological mechanisms between different phenotypes. ${ }^{5}$ Furthermore, TD patients usually show lower striatal dopamine depletion compared to RA patients on [ $\left.{ }^{\mathbf{1 2 3}} \mid\right] \mathrm{FP}$ CIT-SPECT, ${ }^{22}$ suggesting a less pronounced predisposition to LID. Nigrostriatal dopamine depletion is one of the main prerequisite
Table 3. Cox regression analysis of the joint effect of multiple risk factors

\begin{tabular}{llll}
\hline Variable & $\mathrm{HR}$ & $\mathrm{HR} 95 \% \mathrm{Cl}$ & $\mathrm{P}$-value \\
\hline Gender (female) & 1.61 & $1.05-2.47$ & 0.030 \\
$1000 \mathrm{mg} / \mathrm{d}$ of Levodopa LEDD & 1.26 & $1.12-1.42$ & $<0.001$ \\
PIGD/Intermediate vs. TD & 1.95 & $1.28-2.96$ & 0.003 \\
MDS-UPDRS part III & 1.03 & $1.00-1.05$ & 0.032 \\
$\begin{array}{l}\text { Modified Schwab and England ADL } \\
<90\end{array}$ & 1.81 & $0.98-3.38$ & 0.061 \\
$\begin{array}{l}\text { Genetic risk score } \\
\text { Caudate asymmetry Index }\end{array}$ & 1.39 & $1.08-1.78$ & 0.010 \\
$\begin{array}{l}\text { State-Trait Anxiety Inventory - Trait } \\
\text { subscore }\end{array}$ & 1.02 & $1.00-1.03$ & 0.037 \\
\hline
\end{tabular}

Hazard ratios with $95 \%$ confidence intervals and $p$-values

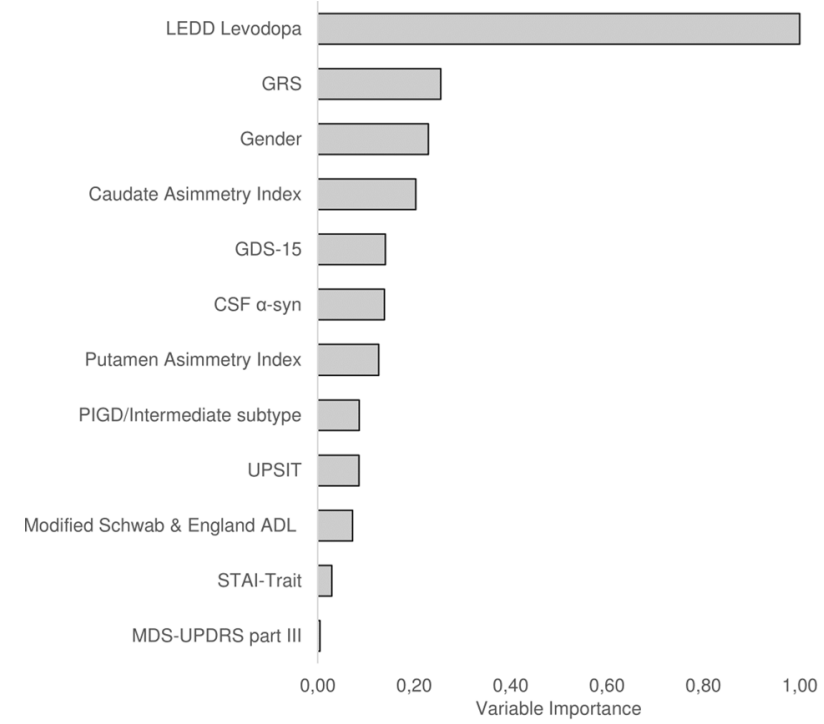

Fig. 2 Ranking of variables according to relative importance in random survival forest model

for developing dyskinesia and dopaminergic denervation is increased by disease severity. Therefore, it is not surprising that disease severity at baseline represents an important predictive factor for LID. Our findings indicate that UPDRS Part III score in denovo patients is a significant clinical biomarker to predict dyskinesia. However, it is not fully consistent with data from STRIDE-PD trial which showed UPDRS Part II score as risk factor for dyskinesia, whereas UPDRS Part III did not correlate with dyskinesia development. ${ }^{19}$ Such contrasting results may be due to the fact that UPDRS motor score does not always reflect precisely the status of presynaptic dopamine denervation evaluated by PET or SPECT. ${ }^{23}$ The relationship between the severity of disease and the susceptibility to dyskinesia is also supported by the significant inverse correlation we found between ADL Scale score and risk of LID. Thus, patients with higher disease severity testified by greater impairment on daily living activities are more prone to develop dyskinesia. Genetic susceptibility to dyskinesia is of great interest, with conflicting results deriving from several studies focusing on single-nucleotide polymorphisms (SNPs) of different genes. PPMI polygenic risk score was developed to explain the risk of idiopathic PD onset so far and it does not include several genes that are known to increase the risk of developing LID. ${ }^{24}$ However, to the best of our 
knowledge, for the first time in this study we highlighted a correlation between cumulative effect of known genetic risk variants of PD and LID development. Such results, if corroborated by extensive genome-wide association studies (GWAS), might represent a focus for future research. Interestingly, we also found that anxiety was associated to an increased risk of dyskinesia. This could be linked to the relationship between dopaminergic dysfunction and neuropsychiatric symptoms in early PD. ${ }^{25}$

Although CSF biomarkers did not explain LID onset in the Cox regression, we found that a-syn ranked 6th, according to the variable importance criterion, in the random survival forests model. This suggest that further investigations are warranted for exploring the role of CSF a-syn in predicting LID, with special attention to pay for the observation in larger cohorts or the measurement of oligomeric and phosphorylated forms.

There are limitations in our data, mainly due to the evaluation of dyskinesia. Even if specific scales have been validated to assess dyskinesia in $\mathrm{PD}^{26}$ they were not used for evaluating qualitative and quantitative aspects of dyskinesia in the PPMI. Therefore, our results neither identified predictive factors for different types of dyskinetic complication in PD (chorea versus dystonia) nor found a possible explanation for the severity of dyskinesia.

In summary, our findings indicate that data deriving from a large cohort of de-novo PD patients monitored longitudinally are useful in understanding the composite aspects involved in the progression of disease. Our results highlight the role of several factors in determining dyskinesia, thus providing useful information for future design of both biomarker studies and randomized clinical trials.

\section{METHODS}

Study design

Overall, 423 de novo PD participants were enrolled in the PPMI study between January 2011 and December 2012. Data were obtained from the PPMI database accessed 30 December 2017.

\section{Participants}

The inclusion criteria for entering PPMI were: (i) age $>30$; (ii) presence of at least two parkinsonian signs such as bradykinesia, rigidity and resting tremor or have an asymmetric resting tremor, or asymmetric bradykinesia; (iii) having received the diagnosis not earlier than two years before enrollment; (iv) documented reduced striatal 123-I loflupane dopamine transporter (DatScan, GE Healthcare, Arlington Heights, IL) imaging binding consistent with PD; (v) no ongoing symptomatic therapy. Each PPMI participant received extensive assessment of motor and non-motor features.

Standard protocol approvals, registrations, and consents

Each participating PPMI site received approval from an ethical standards committee on human experimentation before study initiation. Written informed consent for research was obtained from all individuals participating in the study.

\section{Baseline features}

With the aim of defining predictive factors od LID development among de novo patients, we have investigated several variables available at baseline, including: (i) demographics (age, gender, family history, disease duration, education years); (ii) bradykinesia, rigidity and tremor were assessed according to UK Parkinson's Disease Society Brain Bank Criteria (iii) motor features (International Parkinson's disease and Movement Disorder Society-Unified Parkinson's Disease Rating Scale (MDS-UPDRS)-Part II and III, total tremor score, postural instability-gait disturbance (PIGD) score, tremor/PIGD motor phenotype, Schwab-England activities of daily living (ADL) score, bradykinesia, tremor, and (iv) age/education adjusted Montreal Cognitive Assessment (MoCA); (v) non-motor manifestations (MDS-UPDRS-Part I), olfactory dysfunction via University of Pennsylvania Smell Identification Test (UPSIT) score, autonomic dysfunction via Scale for Outcomes in Parkinson's disease-Autonomic (SCOPA-AUT) total score, REM sleep behaviour disorder (RBD) via RBD screening questionnaire (RBDSQ) score, sleep disturbances via Epworth Sleepiness Score (ESS), anxiety via State-Trait Anxiety Inventory scores (STAI), depression via Geriatric Depression Scale (GDS-15); (vi) genetic risk score including 28 independent risk variants for PD that have been selected according to the results of a meta-analysis of PD genome-wide association studies, ${ }^{24}$ also including $\mathrm{p}$. N370S in GBA and p.G2019S in LRRK2 ${ }^{27,28}$; (vii) CSF biomarkers (CSF amyloid- $\beta 1-42$, total ( $\mathrm{t}$-tau, and phosphorylated tau (P-tau181) and asynuclein); (viii) dopamine transporter imaging striatal-binding ratios (DATscan) (single-photon emission computed tomography (SPECT) with the DAT tracer 1231-ioflupane at baseline, with striatal-binding ratio calculated for left and right putamen separately using the occipital lobe as reference, obtaining ipsilateral, contralateral, mean measurements and asymmetry indices). The asymmetry index for caudate and putamen was calculated, according to PPMI indications for deriving variables, as the difference between left and right divided by the mean value.

\section{Levodopa exposure}

Levodopa equivalent daily dose (LEDD) was reported for each participant reporting the initiation of dopaminergic therapy. We included total LEDD as the cumulative exposure to all dopaminergic drugs, as well as levodopa LEDD as the cumulative exposure to levodopa until the onset of dyskinesia (observed events) or the study exit (censored events).

\section{Levodopa-induced dyskinesia}

The primary outcome was the incidence of LID, defined as the first time the patient reported a positive score in the item "Time spent with dyskinesia" of the MDS-UPDRS part IV.

\section{Statistical analysis}

Statistical analyses were performed using $R$ software version 3.4. Continuous variables were described by means and standard deviations, while categorical ones were reported as count and percentages. Predictors of LID onset were assessed using multivariate Cox proportional hazards regression models. In the absence of a conversion event, data were censored at the most recent clinic visit. We have first assessed the role of each risk factor in conjunction with the total LEDD. In order to avoid rejection of potentially important variables due to uncontrolled confounders, a p-value lower than 0.20 was used as screening criterion to consider the risk factor as candidate for the multivariate analysis. Backward elimination based on the Akaike's information criterion was used to select a final model. Hazard proportionality was assessed through analysis of scaled Schoenfeld residuals whereas martingale residuals were plotted against continuous covariates to detect nonlinearity. As a complementary approach, we have applied random survival forests, ${ }^{29}$ a machine learning technique to determine important variables for the prediction of individual survival times. Variable selection was carried out using a conservative approach based on minimal depth criterion. Significance level of $5 \%$ was assumed for all the analyses.

\section{DATA AVAILABILITY}

Data used in the preparation of this study were obtained from the PPMI database (www.ppmi-info.org/data). For up-to-date information on the study, visit http://www. ppmi-info.org.

\section{ACKNOWLEDGEMENTS}

This study was supported by the Italian Ministry of Health (Grant GR-2013-02357757). The Movement Disorders Center of the University of Perugia was supported by a grant from New York University School of Medicine and The Marlene and Paolo Fresco Institute for Parkinson's and Movement Disorders, which was made possible with support from Marlene and Paolo Fresco.

\section{AUTHOR CONTRIBUTIONS}

P.E., M.R., F.P., P.C., and L.P. conceived the idea, planned and designed the study. P.E., M.R., and F.P. wrote the first draft; P.E. planned the data management and statistical analysis; N.T. provided critical insights. P.E., M.R., F.P., N.T., P.C., and L.P. have approved and contributed to the final written manuscript. 


\section{ADDITIONAL INFORMATION}

Competing interests: The Authors declare no competing interests.

Publisher's note: Springer Nature remains neutral with regard to jurisdictional claims in published maps and institutional affiliations.

\section{REFERENCES}

1. Fereshtehnejad, S. M., Zeighami, Y., Dagher, A. \& Postuma, R. B. Clinical criteria for subtyping Parkinson's disease: biomarkers and longitudinal progression. Brain 140, 1959-1976 (2017).

2. Tambasco, N., Romoli, M. \& Calabresi, P. Levodopa in Parkinson's disease: current status and future developments. Curr. Neuropharmacol. 15, 1-13 (2017).

3. Fahn, S. et al. Levodopa and the progression of Parkinson's disease. N. Engl. J. Med. 351, 2498-508 (2004).

4. Abbott, A. Levodopa: the story so far. Nature 466, S6-S7 (2010).

5. Calabresi, P. et al. Levodopa-induced dyskinesias in patients with Parkinson's disease: filling the bench-to-bedside gap. Lancet Neurol. 9, 1106-1117 (2010).

6. Picconi, B., Hernández, L. F., Obeso, J. A. \& Calabresi, P. Motor complications in Parkinson's disease: striatal molecular and electrophysiological mechanisms of dyskinesias. Mov. Disord. 33, 867-876 (2018).

7. Grandas, F., Galiano, M. L. \& Tabernero, C. Risk factors for levodopa-induced dyskinesias in Parkinson's disease. J. Neurol. 246, 1127-1133 (1999).

8. Fahn, S. Levodopa in the treatment of Parkinson's disease. J. Neural Transm. 71, 1-15 (2006).

9. Schrag, A. \& Quinn, N. Dyskinesias and motor fluctuations in Parkinson's disease. Brain 123, 2297-2305 (2000).

10. Zappia, M. et al. Sex differences in clinical and genetic determinants of levodopa peak-dose dyskinesias in Parkinson disease. Arch. Neurol. 62, 601 (2005).

11. Arabia, G. et al. Body weight, levodopa pharmacokinetics and dyskinesia in Parkinson's disease. Neurol. Sci. 23, 53-54 (2002).

12. Yoo, H. S. et al. Presynaptic dopamine depletion determines the timing of levodopa-induced dyskinesia onset in Parkinson's disease. Eur. J. Nucl. Med. Mol. Imaging 1-9 (2017). https://doi.org/10.1007/s00259-017-3844-8

13. Hong, J. Y. et al. Presynaptic dopamine depletion predicts levodopa-induced dyskinesia in de novo Parkinson disease. Neurology 82, 1597-1604 (2014).

14. Linazasoro, G. et al. Levodopa-induced dyskinesias in parkinson disease are independent of the extent of striatal dopaminergic denervation: a pharmacological and SPECT study. Clin. Neuropharmacol. 32, 326-329 (2009).

15. De La Fuente-Fernndez, R. et al. Biochemical variations in the synaptic level of dopamine precede motor fluctuations in Parkinson's disease: PET evidence of increased dopamine turnover. Ann. Neurol. 49, 298-303 (2001).

16. Sharma, J. C., Bachmann, C. G. \& Linazasoro, G. Classifying risk factors for dyskinesia in Parkinson's disease. Park. Relat. Disord. 16, 490-497 (2010).

17. Blanchet, P. J. et al. Short-term effects of high-dose 17 beta-estradiol in postmenopausal PD patients: a crossover study. Neurology 53, 91-95 (1999).
18. Penney, J. B. et al. Impact of deprenyl and tocopherol treatment on Parkinson's disease in DATATOP patients requiring levodopa. Ann. Neurol. 39, 37-45 (1996).

19. Warren Olanow, C. et al. Factors predictive of the development of Levodopainduced dyskinesia and wearing-off in Parkinson's disease. Mov. Disord. 28, 1064-1071 (2013).

20. Contrafatto, D. et al. Single photon emission computed tomography striatal asymmetry index may predict dopaminergic responsiveness in Parkinson disease. Clin. Neuropharmacol. 34, 71-3 (2011).

21. Kipfer, S., Stephan, M. A., Schüpbach, W. M. M., Ballinari, P. \& Kaelin-Lang, A. Resting tremor in Parkinson disease: a negative predictor of levodopa-induced dyskinesia. Arch. Neurol. 68, 1037-1039 (2011).

22. Rossi, $C$. et al. Differences in nigro-striatal impairment in clinical variants of early Parkinson's disease: evidence from a FP-CIT SPECT study. Eur. J. Neurol. 17, 626-630 (2010).

23. Benamer, H. T. S. et al. Correlation of Parkinson's disease severity and duration with 123I-FP-CIT SPECT striatal uptake. Mov. Disord. 15, 692-698 (2000).

24. Nalls, M. A. et al. Large-scale meta-analysis of genome-wide association data identifies six new risk loci for Parkinson's disease. Nat. Genet. 46, 989-993 (2014).

25. Picillo, M. et al. Association between dopaminergic dysfunction and anxiety in de novo Parkinson's disease. Park. Relat. Disord. 37, 106-110 (2017).

26. Colosimo, C. et al. Task force report on scales to assess dyskinesia in Parkinson's disease: critique and recommendations. Mov. Disord. 25, 1131-1142 (2010).

27. International Parkinson Disease Genomics Consortium et al. Imputation of sequence variants for identification of genetic risks for Parkinson's disease: a meta-analysis of genome-wide association studies. Lancet 377, 641-9 (2011).

28. Nalls, M. A. et al. Baseline genetic associations in the Parkinson's Progression Markers Initiative (PPMI). Mov. Disord. 31, 79-85 (2016).

29. Ishwaran, H., Kogalur, U. B., Blackstone, E. H. \& Lauer, M. S. Random survival forests. Ann. Appl. Stat. 2, 841-860 (2008).

(C) Open Access This article is licensed under a Creative Commons Attribution 4.0 International License, which permits use, sharing, adaptation, distribution and reproduction in any medium or format, as long as you give appropriate credit to the original author(s) and the source, provide a link to the Creative Commons license, and indicate if changes were made. The images or other third party material in this article are included in the article's Creative Commons license, unless indicated otherwise in a credit line to the material. If material is not included in the article's Creative Commons license and your intended use is not permitted by statutory regulation or exceeds the permitted use, you will need to obtain permission directly from the copyright holder. To view a copy of this license, visit http://creativecommons. org/licenses/by/4.0/.

(c) The Author(s) 2018 